\title{
Light Transmission and the Sagnac Effect on the Rotating Earth
}

\author{
Stephan J. G. Gift ${ }^{1}$ \\ ${ }^{1}$ Department of Electrical and Computer Engineering, Faculty of Engineering, The University of the West Indies, \\ St. Augustine, Trinidad and Tobago, West Indies \\ Correspondence: Stephan J. G. Gift, Department of Electrical and Computer Engineering, Faculty of Engineering, \\ The University of the West Indies, St. Augustine, Trinidad and Tobago, West Indies. E-mail: \\ Stephan.Gift@sta.uwi.edu
}

Received: June 9, 2013 Accepted: July 3, 2013 Online Published: September 3, 2013

doi:10.5539/apr.v5n5p93 URL: http://dx.doi.org/10.5539/apr.v5n5p93

\begin{abstract}
Light transmission on the surface of the rotating Earth is examined using the Langevin metric of general relativity. It is shown that this metric which yields the rigorously tested and verified algorithm for clock synchronization in the Global Positioning System (GPS) also predicts east-west light speed anisotropy that is inconsistent with light speed isotropy postulated to hold at a local level on the rotating Earth. This light speed anisotropy is further demonstrated using experimentally confirmed light travel time in a simple speed calculation as well as the classical method of light speed relative to a terrestrial observer rotating with the Earth for light travel in the Earth-Centred Inertial (ECI) frame. These theoretical results are confirmed by experimental findings from GPS measurements and lead to the re-interpretation of the Sagnac effect normally treated as a time correction in GPS operation.
\end{abstract}

Keywords: general relativity, principle of light speed constancy, GPS, range equation, one-way speed of light, ECI frame, Sagnac Effect

\section{Introduction}

A central tenet of modern physics is the principle of light speed constancy according to which light travels at a constant speed $c$ in all inertial frames. Numerous verification experiments yielding light speed $c$ have been conducted leading to the almost universal belief that the principle has been confirmed. However Zhang (1997) has shown that while two-way light speed invariance has been verified, one-way light speed constancy has not. Also, although the principle is formulated as applying in inertial frames, the majority of light speed tests claiming its confirmation have been conducted in the non-inertial frame of the rotating Earth (Brillet \& Hall, 1979; Gagnon et al., 1988; Riis et al., 1988; Krisher et al., 1990; Muller et al., 2003; Hermann et al., 2005; Antonini et al., 2005; Eisele et al., 2009). Rindler (2006) justifies this by arguing “...we do not expect to find such extended or perfect inertial frames in nature. It is the equivalence principle that provides the bridge between the ideal [special relativity] model and the real world. According to it, we can find at each event a set of local inertial frames, which may be small or large depending on (i) the distribution of nearby masses, and (ii) the accuracy we require. It is to these frames (or other frames not differing too much from these frames) that [special relativity] is applied in practice, and with great success." In other words the surface of the rotating Earth can be treated as an approximately inertial frame by confining considerations to suitably restricted regions such that the speed of light is locally isotropic in this otherwise non-inertial frame. This conclusion is supported by many authors including Rizzi and Tartaglia (1998, 1999), Rizzi and Ruggiero (2002), Pascual-Sánchez et al. (2010) and Kassner (2012). Thus on a rotating platform Rizzi and Tartaglia (1998) argue for light speed constancy both East and West and Pascual-Sánchez et al. (2010) support light speed constancy both locally and globally. Additionally, Tartaglia and Ruggiero (2002) have shown in the framework of general relativity that angular momentum effects do not measurably affect light travel times in Michelson-Morley type experiments. Perhaps the most compelling indication of the acceptance of the principle of light speed constancy and its applicability in the terrestrial frame is its use in the SI definition of the metre requiring light speed invariance in the frame of the rotating Earth.

There is however some disagreement about the physics of light transmission on a rotating platform. Specifically a few authors argue for light speed anisotropy around such a platform and the consequent inapplicability of the principle of light speed constancy (Selleri, 1997; Klauber, 1999). Selleri (1997) in particular advanced a proof of 
anisotropy in the speed of light in a reference frame that is comoving with the edge of a rotating platform. Even Rizzi and Tartaglia (1998) who initially argued for light speed isotropy, later relaxed their position regarding global light speed anisotropy in a rotating frame (Rizzi \& Tartaglia, 1999). An entire book addressing this issue has in fact been published (Rizzi \& Ruggiero, 2010). Thirty years ago in a paper presented at a Precise Time and Time Interval meeting describing the results of time transfer experiments using laser light pulses, Alley and others (Alley et al., 1982) noted that the metric associated with general relativity predicts an asymmetry in the one-way speed of light travelling in the east-west and west-east directions. It was suggested then that this difference if real may eventually be measurable by experiment. In a later paper Alley and his colleagues (Alley et al., 1988) outlined the general relativity theory prediction of light speed anisotropy in the east-west direction and described an experiment that directly searched for this anisotropy. This prediction of light speed anisotropy in the framework of general relativity directly conflicts with the local principle of light speed constancy on the surface of the Earth and this apparent inconsistency has never been resolved.

The advent of the very successful Global Positioning System (GPS) where light transmission on the rotating Earth is an on-going physical activity provides an opportunity for direct experimental resolution of the controversy relating to the physics of light travelling in rotating frames. GPS operation is based on accurate atomic clocks that are synchronized using an algorithm derived within the same general relativity metric (IS-GPS-200E, 2010; Ashby, 2003) that predicts east-west light speed asymmetry. The accepted interpretation is that light speed is constant in GPS clock synchronization but a time adjustment sometimes referred to as the "Sagnac correction" is necessary because of the Earth's rotation. Similar to the case of light speed constancy in a rotating frame, this interpretation has been subject to challenge (Marmet, 2000; Kelly, 2005; Gift, 2010, 2011; Wang, 2000; Hatch, 2007; Sato, 2010). Marmet (2000) and Kelley (2005) have argued that observed travel time differences in the GPS for east-west light transmission is evidence of light speed anisotropy and Gift has supported this using the rigorously verified GPS clock synchronization algorithm (Gift, 2010) and the GPS range equation (Gift, 2011). Hayden (1991) previously arrived at this same conclusion after considering several experiments including Sagnac, Michelson-Gale and Brillet-Hall.

In this paper we re-examine the issue of light transmission on the rotating Earth with particular reference to the GPS and analyze the problem using general relativity in a rotating frame of reference. In section 2 we derive the transmission time for a pulse of light travelling on the surface of the Earth using the Langevin metric in general relativity. This yields the result given by Ashby and accepted by the CCIR for clock synchronization. In section 3 we use the same metric to determine the corresponding east-west light speeds on the rotating Earth and revisit the problem of asymmetrical light speeds. Supporting experimental evidence from the GPS is presented. In section 4 based on the experimentally confirmed time of travel result light speed in any direction on the surface of the Earth is calculated using the ratio distance over time. In section 5 light speed in any direction is also determined based on light travel in the Earth-Centered Inertial (ECI) frame relative to the rotating Earth. A full discussion of the findings and their implications is then presented in section 6 .

\section{Derivation of the Travel Time of a Light Pulse in General Relativity}

In this section we derive the transmission time of a light pulse travelling on the surface of the Earth. In doing so, we follow the procedure presented by Ashby (2003). From general relativity and ignoring gravitational potentials, the metric in the ECI frame where space-time is Minkowskian is in cylindrical coordinates given by (Landau \& Lifschitz, 1975; Moller, 1972; Adler et al., 1975)

$$
-d s^{2}=-(c d t)^{2}+d r^{2}+r^{2} d \varphi^{2}+d z^{2}
$$

The transformation from the coordinate system $(t, r, \varphi, z)$ in the ECI frame where light speed is $c$ to a coordinate system $\left(t^{\prime}, r^{\prime}, \varphi^{\prime}, z^{\prime}\right)$ in the Earth Centered Earth Fixed frame which is rotating at the uniform angular speed $\omega_{E}$ is given by

$$
t=t^{\prime}, \quad r=r^{\prime}, \varphi=\varphi^{\prime}+\omega_{E} t^{\prime}, \quad z=z^{\prime}
$$

The transformation $t=t^{\prime}$ in (2) means that time $t^{\prime}$ in the rotating frame is the same as the time $t$ in the underlying ECI frame and this can be achieved by appropriate clock synchronization. This results in the Langevin metric in the rotating frame given by

$$
-d s^{2}=-\left(1-\frac{\omega_{E}{ }^{2} r^{\prime 2}}{c^{2}}\right)\left(c d t^{\prime}\right)^{2}+2 \omega_{E} r^{\prime 2} d \varphi^{\prime} d t^{\prime}+d \sigma^{\prime 2}
$$

where 


$$
d \sigma^{\prime 2}=d r^{2}+r^{\prime 2} d \varphi^{\prime 2}+d z^{2}
$$

is the square of the coordinate distance. Applying Equation (3) to the propagation of light on the rotating Earth by setting $d s^{2}=0$ yields a quadratic equation for $d t^{\prime}$ given by

$$
\left(1-\frac{\omega_{E}{ }^{2} r^{\prime 2}}{c^{2}}\right)\left(c d t^{\prime}\right)^{2}-\frac{2 \omega_{E} r^{\prime 2} d \varphi^{\prime}}{c}\left(c d t^{\prime}\right)-d \sigma^{\prime 2}=0
$$

Solving for $\left(c d t^{\prime}\right)$ gives

$$
c d t^{\prime}=\frac{\frac{2 \omega_{E} r^{\prime 2} d \varphi^{\prime}}{c} \pm \sqrt{\left(\frac{2 \omega_{E} r^{\prime 2} d \varphi^{\prime}}{c}\right)^{2}+4\left(1-\frac{\omega_{E}{ }^{2} r^{\prime 2}}{c^{2}}\right) d \sigma^{\prime 2}}}{2\left(1-\frac{\omega_{E}{ }^{2} r^{\prime 2}}{c^{2}}\right)}
$$

Taking the dominant term under the square root gives

$$
c d t^{\prime}=\frac{\frac{\omega_{E} r^{\prime 2} d \varphi^{\prime}}{c} \pm d \sigma^{\prime}}{\left(1-\frac{\omega_{E}{ }^{2} r^{\prime 2}}{c^{2}}\right)}
$$

Noting that $\frac{\omega_{E}{ }^{2} r^{\prime 2}}{c^{2}}<<1$ and taking the positive sign reduce Equation (7) to

$$
c d t^{\prime}=\frac{\omega_{E} r^{\prime 2} d \varphi^{\prime}}{c}+d \sigma^{\prime}
$$

As pointed out by Ashby (2003) the quantity $r^{\prime 2} d \varphi^{\prime} / 2$ corresponds to the infinitesimal area $d A_{Z}^{\prime}$ in the rotating frame that is swept out by a line connecting the axis of rotation to the light pulse and projected onto a plane parallel to the equatorial plane. Thus (8) can be written as

$$
d t^{\prime}=\frac{d \sigma^{\prime}}{c}+\frac{2 \omega_{E}}{c^{2}} d A_{z}^{\prime}
$$

From (9) the total time for light to travel a given path as measured in the rotating frame is given by

$$
\int_{\text {path }} d t^{\prime}=\int_{\text {path }} \frac{d \sigma^{\prime}}{c}+\frac{2 \omega_{E}}{c^{2}} \int_{\text {path }} d A_{z}^{\prime}
$$

Equation (10) gives the light travel time observed on the surface of the rotating Earth. As a GPS clock synchronization procedure it has been rigorously and exhaustively tested and verified and has been published in a standard by the CCIR (International Radio Consultative Committee) in 1990 and 1997 for synchronizing clocks at different points on the Earth. Using (10) the time $\Delta t_{W E}$ for light to travel eastward between two points fixed on the surface of the Earth a short distance $l$ apart is given by (Gift, 2010)

$$
\Delta t_{W E}=\frac{l}{c}+\frac{l v}{c^{2}}
$$

where $v$ is the surface speed of the Earth at the particular latitude. The time $\Delta t_{E W}$ for light to travel westward between these same two points from (10) is given by (Gift, 2010)

$$
\Delta t_{E W}=\frac{l}{c}-\frac{l v}{c^{2}}
$$

This GPS east-west light travel time differential $\Delta t_{W E}-\Delta t_{E W}>0$ derived using the CCIR algorithm (10) formally establishes the fact that light takes longer to travel East than West between fixed points on the surface of the Earth, an experimental observation first highlighted by Marmet (2000) and Kelley (2005).

Ashby however interprets the first term $\int d \sigma^{\prime} / c$ in (10) as the time in the rotating frame for the light to traverse the path $d \sigma^{\prime}$ at speed $c$ while he interprets the second term $\frac{2 \omega_{E}}{c^{2}} \int d A_{z}^{\prime}$ as the additional time determined in the underlying inertial frame for the light "to catch up to the moving reference point". The associated physical 
phenomenon is sometimes referred to as the Sagnac Effect. According to Ashby "Observers fixed on the Earth, who were unaware of Earth rotation, would use just $\int d \sigma^{\prime} / c$ for synchronizing their clock network" and this would lead to significant error. Towards accurate clock synchronization he states that corrections of the form $2 \omega_{E} \int_{\text {path }} d A_{z}^{\prime} / c^{2}$ must be applied, this based on his interpretation of Equation (10).

From this discussion, the overall situation for the observer fixed on the surface of the rotating Earth is that light travels between fixed points on the Earth's surface at speed $c$ consistent with the principle of light speed constancy but that a "Sagnac correction" must be applied to the resulting travel time because of the rotation of the Earth. Ashby has published several papers promoting this interpretation (Ashby, 2002, 2003, 2006, 2010) and it is now widely accepted by physicists. This interpretation is however open to question since as indicated previously the Langevin metric used to derive the clock synchronization algorithm also yields anisotropic light speed in the east-west direction in the rotating frame (Alley et al., 1982, 1988). Moreover as demonstrated above, Equation (10) shows that $\Delta t_{W E} \neq \Delta t_{E W}$ implying asymmetrical east-west light speeds. We examine this situation in the following sections.

\section{Light Speed in the East-West Direction in General Relativity}

Following Alley et al. (1988), consider light transmission in the east-west direction on the rotating Earth as shown in Figure 1 in which case $d z^{\prime}=0$. These authors assumed $d r^{\prime}=0$ in their derivation of east-west light speed since the changes are incremental. In order to demonstrate this, starting in the direction of a line of latitude $O B$, the light pulse travels from point $O$ on the surface of the Earth along a straight line to the point $A$ where $B$ is on the surface of the Earth and $O^{\prime}$ is on the axis. For right-angled triangle $O^{\prime} O A, d \sigma^{\prime}=r^{\prime} t a n d \varphi^{\prime} \approx r^{\prime} d \varphi^{\prime}$ since $d \varphi^{\prime}$ is an infinitesimal change and hence from (4), $d r^{\prime}=0$. Using $d \sigma^{\prime} \approx r^{\prime} d \varphi^{\prime}$ reduces Equation (7) to

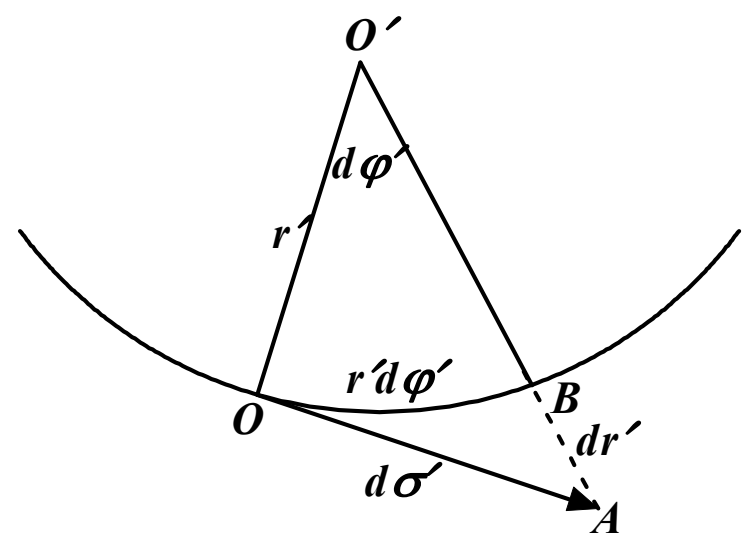

Figure 1. East-West light transmission on the surface of the Earth

$$
c d t^{\prime}=\frac{\frac{\omega_{E} r^{\prime 2} d \varphi^{\prime}}{c} \pm r^{\prime} d \varphi^{\prime}}{\left(1-\frac{\omega_{E}^{2} r^{\prime 2}}{c^{2}}\right)}=\frac{r^{\prime} d \varphi^{\prime}\left(\frac{\omega_{E} r^{\prime}}{c} \pm 1\right)}{\left(1+\frac{\omega_{E} r^{\prime}}{c}\right)\left(1-\frac{\omega_{E} r^{\prime}}{c}\right)}
$$

For light travelling in the east-west direction, $d \varphi^{\prime}$ is negative such that $d \varphi^{\prime}=-\left|d \varphi^{\prime}\right|$. Since $d t^{\prime}$ is positive, then the negative sign in $\left(\frac{\omega_{E} r^{\prime}}{c^{2}} \pm 1\right)$ in (13) is chosen giving

$$
c d t^{\prime}=\frac{r^{\prime}\left|d \varphi^{\prime}\right|\left(1-\frac{\omega_{E} r^{\prime}}{c}\right)}{\left(1+\frac{\omega_{E} r^{\prime}}{c}\right)\left(1-\frac{\omega_{E} r^{\prime}}{c}\right)}=\frac{r^{\prime}\left|d \varphi^{\prime}\right|}{\left(1+\frac{\omega_{E} r^{\prime}}{c}\right)}
$$

This gives 


$$
d t^{\prime}=\frac{r^{\prime}\left|d \varphi^{\prime}\right|}{\left(c+\omega_{E} r^{\prime}\right)}
$$

Now $r^{\prime}\left|d \varphi^{\prime}\right|=d$ is the east-west distance travelled in time $d t^{\prime}$ and $\omega_{E} r^{\prime}=v$ is the speed of the Earth's surface at the particular latitude. Hence

$$
d t^{\prime}=\frac{d}{(c+v)}
$$

Therefore the east-west light speed $c_{E W}$ on the rotating Earth is given by

$$
c_{E W}=\frac{d}{d t^{\prime}}=\frac{d}{d /(c+v)}=c+v
$$

For light travelling in the west-east direction, $d \varphi^{\prime}$ is positive and therefore $d \varphi^{\prime}=\left|d \varphi^{\prime}\right|$. Since $d t^{\prime}$ is positive, then the positive sign in $\left(\frac{\omega_{E} r^{\prime}}{c^{2}} \pm 1\right)$ in (13) is chosen giving

$$
c d t^{\prime}=\frac{r^{\prime}\left|d \varphi^{\prime}\right|\left(1+\frac{\omega_{E} r^{\prime}}{c}\right)}{\left(1+\frac{\omega_{E} r^{\prime}}{c}\right)\left(1-\frac{\omega_{E} r^{\prime}}{c}\right)}=\frac{r^{\prime}\left|d \varphi^{\prime}\right|}{\left(1-\frac{\omega_{E} r^{\prime}}{c}\right)}
$$

This reduces to

$$
d t^{\prime}=\frac{r^{\prime}\left|d \varphi^{\prime}\right|}{\left(c-\omega_{E} r^{\prime}\right)}
$$

which gives

$$
d t^{\prime}=\frac{d}{(c-v)}
$$

Therefore the west-east light speed $c_{W E}$ on the rotating Earth is given by

$$
c_{W E}=\frac{d}{d t^{\prime}}=\frac{d}{d /(c-v)}=c-v
$$

Thus at any point on the surface of the rotating Earth, the general relativity calculation produces light speed $c+v$ from East to West and light speed $c-v$ from West to East. Alley et al., (1988) noted this asymmetrical light speed prediction by the general relativity metric but were unclear whether the prediction was observable since the metric of Equation (3) "seems to assume that the observer is located at the centre of the rotation." This uncertainty has however been removed as light speeds $c \pm v$ in the east-west direction on the surface of the Earth have been verified by several researchers using GPS technology.

Around the time of these attempts by Alley and his group to detect East-West light speed differentials, unequal travel time of about 300 nanoseconds for electromagnetic signals travelling in opposite directions around the Earth was directly demonstrated by Allan and his colleagues using atomic clocks and signal reflections off orbiting satellites (Allan et al., 1985). Following this Marmet (2000), using GPS measurements observed that a light signal takes about 28 nanoseconds longer to travel eastward from San Francisco to New York than to travel westward from New York to San Francisco. Kelly (2005) also noted that measurements using the GPS reveal that a light signal takes 414 nanoseconds longer to circumnavigate the Earth eastward at the equator than in the westward direction around the same path. This is as predicted by GPS Equations (11) and (12). Both Marmet and Kelley concluded that these observed travel time differences in the GPS measurements in each direction occur because light travels at speed $c-v$ eastward and $c+v$ westward relative to the surface of the Earth at the particular latitude. Gift (2010) confirmed and generalized these anisotropic east-west light speeds $c \pm v$ by using the GPS clock synchronization algorithm to determine light travel time for arbitrary distance. (See section 4)

Wang (2000) used the range equation operating in the ECI frame to demonstrate light travel time differences depending on the observer's uniform motion relative to the ECI frame. Gift (2011) applied Wang's approach by employing the range equation to determine elapsed time for light traveling between two adjacent points fixed on the surface of the rotating Earth at the same latitude then using the known distance between the two fixed points 
to show that the one-way speed of light in the east-west direction is $c \pm v$. This east-west light speed anisotropy has also been observed by Hatch (2007) for light transmission between the GPS receivers on two Gravity Recovery and Climate Experiment satellites and by Sato (2010) who showed that in the operation of the GPS the Sagnac effect provides experimental evidence that the speed of light varies according to the rotational motion of the Earth.

It is evident therefore that the asymmetrical light speed prediction of general relativity has actually been observed, the inconsistency with the principle of light speed constancy notwithstanding. In order for general relativity to predict light speed constancy on a rotating platform, it seems that the associated field equation must be modified. Based on research conducted by Yilmaz $(1976,1982)$ a suitable modification is adding the stress energy tensor of the gravitational field $t_{\mu \nu}$ to the stress energy tensor for matter $T_{\mu \nu}$ on the right side of the equation. The equation then becomes

$$
R_{\mu \nu}-\frac{1}{2} g_{\mu v} R=\frac{8 \pi G}{c^{4}}\left(T_{\mu v}+t_{\mu v}\right)
$$

where $R_{\mu v}$ is the Ricci tensor constructed from a nonlinear combination of the $g_{\mu v}$ and their first and second derivatives, $R$ is the scalar curvature and $G$ is the Newtonian gravitational constant. The relevant metric when solved for east-west light travel yields light speed constancy given by (Alley et al., 1988)

$$
r^{\prime} \frac{d \varphi^{\prime}}{d t^{\prime}}= \pm c
$$

However while light speed $c$ derived using the modified field equation is known to produce inaccurate clock synchronization when used without a "Sagnac correction", it will be shown that light speeds $c \pm v$ derived using the original field equation do not.

\section{Light Speed Using GPS Time Measurements}

Since the time of travel given by GPS Equations (11) and (12) has been rigorously verified and is programmed into the operation of the GPS, this represents an actual time of travel measurement that can be used to test the asymmetrical east-west light speed prediction of general relativity based on simple kinematics as was done by Gift (2010). Thus using the GPS time $\Delta t_{W E}$ in (11), the one-way speed of light $c_{W E}$ traveling eastward between two points fixed on the surface of the Earth a distance $l$ apart is given by

$$
c_{W E}=\frac{l}{\Delta t_{W E}}=\frac{l}{\frac{l}{c}+\frac{l v}{c^{2}}}=c\left(1+\frac{v}{c}\right)^{-1}=c-v, v<<c
$$

Similarly using the GPS time $\Delta t_{E W}$ in (12), the one-way speed of light $c_{E W}$ traveling westward between the two clocks is given by

$$
c_{E W}=\frac{l}{\Delta t_{E W}}=\frac{l}{\frac{l}{c}-\frac{l v}{c^{2}}}=c\left(1-\frac{v}{c}\right)^{-1}=c+v, v<c
$$

The results in (24) and (25) are exactly the light speeds (21) and (17) predicted by general relativity. This means that the GPS directly confirms light speed anisotropy in the Earth Centered Earth Fixed frame. Stated simply, the GPS shows that light travels faster West than East between two points fixed on the surface of the rotating Earth.

It is possible to use the GPS to demonstrate a more general form of the asymmetrical east-west light speed prediction of general relativity. Thus in the rotating frame the light pulse travels a coordinate distance $d \sigma^{\prime}$ in the time $d t^{\prime}$ given in (9) and hence the light speed $c_{R}$ in the rotating frame of the Earth is given by

$$
c_{R}=\frac{d \sigma^{\prime}}{d t^{\prime}}=\frac{d \sigma^{\prime}}{\frac{d \sigma^{\prime}}{c}+\frac{\omega_{E} r^{\prime 2} d \varphi^{\prime}}{c^{2}}}
$$

In Figure 2, $O^{\prime}$ is on the axis of the Earth, $O$ is on the surface and $d A_{Z}^{\prime}$ is parallel to the equatorial plane. Let the angle between the direction of the light pulse on the surface of the Earth and the line of latitude $O X$ at that point be $\theta$. Then

$$
r^{\prime} d \varphi^{\prime}=d \sigma^{\prime} \cos \theta
$$

Recalling that $\omega_{E} r^{\prime}=v$ is the speed of the Earth's surface at the particular latitude and using (27), (26) becomes 


$$
c_{R}=\frac{d \sigma^{\prime}}{\frac{d \sigma^{\prime}}{c}+\frac{v d \sigma^{\prime} \cos \theta}{c^{2}}}
$$

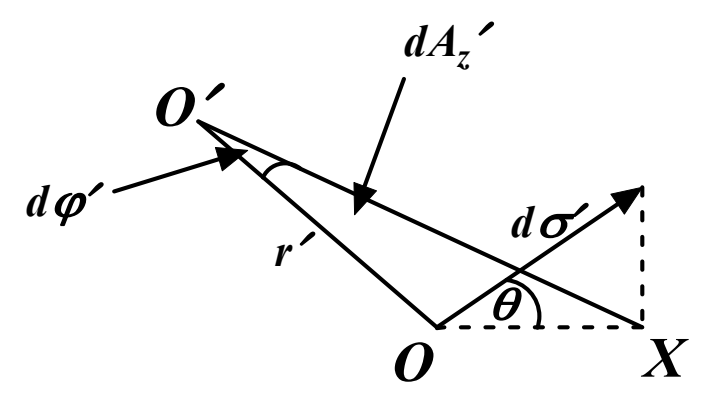

Figure 2. Distance $d \sigma^{\prime}$ travelled by the Light Pulse

This reduces to

$$
c_{R}=c\left(1+\frac{v}{c} \cos \theta\right)^{-1}=c-v \cos \theta, v<<c
$$

For light transmission in the west-east direction, $\theta=0^{\circ}$ and therefore (29) gives $c_{R}=c-v$. For light transmission in the east-west direction, $\theta=180^{\circ}$ and hence (29) yields $c_{R}=c+v$. These light speed values are exactly those found using the general relativity metric in section 3 and experimentally confirmed using the GPS (Marmet, 2000; Kelly, 2005; Gift, 2010; Gift, 2011; Wang, 2000; Hatch, 2007; Sato, 2010).

Thus the light speed in (29) determined in the Earth's rotating frame using the CCIR timing algorithm (9) and (10) yields one-way light speed $c_{R}=c-v \cos \theta$ relative to the surface of the Earth and not light speed $c_{R}=c$ required by the local principle of light speed constancy. It is therefore now clear why clock synchronization using $\int d \sigma^{\prime} / c$ alone results in significant error. The error occurs because light speed in the rotating frame is $c-$ $v \cos \theta$ and not $c$ and hence clock synchronization should use $\int d \sigma^{\prime} /(c-v \cos \theta)$ and not $\int d \sigma^{\prime} / c$. To confirm this we determine the time $d t^{\prime}$ for light to travel the coordinate distance $d \sigma^{\prime}$ at speed $c_{R}=c-v \cos \theta$. This is given by

$$
d t^{\prime}=\frac{d \sigma^{\prime}}{c_{R}}=\frac{d \sigma^{\prime}}{c-v \cos \theta}=\frac{d \sigma^{\prime}(c+v \cos \theta)}{\left(c^{2}-v^{2} \cos ^{2} \theta\right)}=\frac{d \sigma^{\prime}(c+v \cos \theta)}{c^{2}}, v<<c
$$

Then,

$$
d t^{\prime}=\frac{d \sigma^{\prime}}{c}+\frac{d \sigma^{\prime} r^{\prime} \omega_{E} \cos \theta}{c^{2}}
$$

From Figure 2,

Substituting (32) in (31) yields

$$
d \sigma^{\prime} \cos \theta \times r^{\prime} / 2=d A_{z}^{\prime}
$$

$$
d t^{\prime}=\frac{d \sigma^{\prime}}{c}+\frac{2 \omega_{E}}{c^{2}} d A_{z}^{\prime}
$$

From (33) the time for light to travel a given path as measured in the rotating frame is therefore given by

$$
\int_{\text {path }} d t^{\prime}=\int_{\text {path }} \frac{d \sigma^{\prime}}{c}+\frac{2 \omega_{E}}{c^{2}} \int_{\text {path }} d A_{z}^{\prime}
$$

which is Equation (10) given by Ashby and published by the CCIR for clock synchronization. We have therefore confirmed that clock synchronization using $\int d \sigma^{\prime} / c_{R}$ where $c_{R}=c-v \cos \theta$ results in accurate clock synchronization. Ashby's interpretation that synchronization using (34) is really the use of travel time $\int d \sigma^{\prime} / c$ with a "correction" $2 \omega_{E} \int d A_{Z}^{\prime} / c^{2}$ promotes the illusion that the actual speed of light in the rotating frame is $c$ consistent with the local principle of light speed constancy when in fact the speed is $c-v \cos \theta$ as has now been demonstrated. In view of these results Ashby's interpretation must be rejected. 


\section{Derivation of Light Speed Using Relative Velocity in the ECI Frame}

According to the IS-GPS-200E Interface Specification (IS-GPS-200E, 2010), GPS signals propagate in straight lines at the constant speed $c$ in the ECI frame, a frame that moves with the Earth in its orbital motion but does not share the Earth's rotational motion. The experimentally established isotropy of the speed of light in the ECI frame is utilized in the GPS range equation given by (IS-GPS-200E, 2010; Ashby, 2003)

$$
\left|\bar{r}_{r}\left(t_{r}\right)-\bar{r}_{s}\left(t_{s}\right)\right|=c\left(t_{r}-t_{s}\right)
$$

where $t_{s}$ is the time of transmission of an electromagnetic signal from a source, $t_{r}$ is the time of reception of the electromagnetic signal by a receiver both times determined using synchronized clocks, $\bar{r}_{s}\left(t_{s}\right)$ is the position of the source at the time of transmission of the signal and $\bar{r}_{r}\left(t_{r}\right)$ is the position of the receiver at the time of reception of the signal. Equation (35) enables accurate determination of the instantaneous position of objects which are stationary or moving on the surface of the Earth. This light speed constancy in the ECI frame provides us with a third approach to confirming light speed anisotropy in the rotating frame of the surface of the Earth.

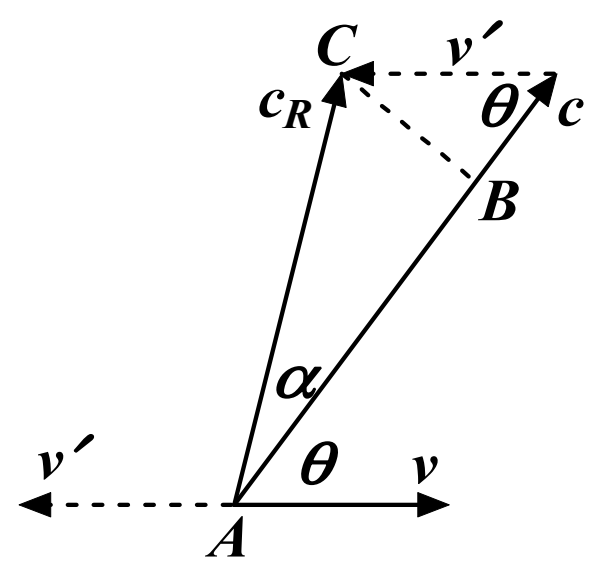

Figure 3. Light speed in the ECI frame and observer speed due to Earth's rotation

Thus in the ECI frame light travels at speed $c$ relative to the ECI frame while the Earth on whose surface the light transmission is observed is rotating at speed $v$ relative to the ECI frame. Consider a light pulse directed at an angle $\theta$ with respect to the line of latitude at a point $\mathrm{A}$ on the Earth. This is shown in Figure 3 where the light is travelling at speed $c$ in the ECI frame and the observer on the surface of the Earth is moving at speed $v$ because of the rotating Earth. In order to determine velocity of the light relative to the moving observer a velocity $v^{\prime}$ that is equal and opposite to $v$ is applied in order to bring the observer to rest relative to the ECI frame. Then the speed of the light $c_{R}$ relative to the rotating Earth is the resultant of $c$ and $v^{\prime}$ given by

$$
c_{R}^{2}=c^{2}+v^{2}+2 v c \cos (180-\theta)=c^{2}+v^{2}-2 v c \cos \theta=(c-v \cos \theta)^{2}+(v \sin \theta)^{2}
$$

Since $v<<$, then (36) reduces to

$$
c_{R}=c-v \cos \theta
$$

which is exactly the speed (29) derived in section (4). Considering the right-angled triangle $A B C$ in Figure $3, A B$ $=(c-v \cos \theta)$ and $B C=v \sin \theta$. Hence the associated angle $\alpha$ is given by

$$
\tan \alpha=\frac{B C}{A B}=\frac{v \sin \theta}{c-v \cos \theta} \approx 0, v<<c
$$

From (38) $\alpha=0$ and therefore the speed of light $c_{R}$ relative to the surface of the rotating Earth is $c_{R}=c-v \cos \theta$ at an angle $\theta$ with the line of latitude. The time $d t^{\prime}$ to travel the coordinate distance $d \sigma^{\prime}$ at speed $c_{R}=c-v \cos \theta$ is given by

$$
d t^{\prime}=\frac{d \sigma^{\prime}}{c_{R}}=\frac{d \sigma^{\prime}(c+v \cos \theta)}{c^{2}}, v<<c
$$

which as shown in section (4) leads to the CCIR synchronization Equation (10) given by 


$$
\int_{\text {path }} d t^{\prime}=\int_{\text {path }} \frac{d \sigma^{\prime}}{c}+\frac{2 \omega_{E}}{c^{2}} \int_{\text {path }} d A_{z}^{\prime}
$$

It is instructive to compare this situation with the case of a vehicle moving uniformly on the surface of the Earth and observed by a pedestrian. In order to have a meaningful comparison we again consider the time $\Delta t_{W E}$ for light to travel eastward between two points fixed on the surface of the Earth a short distance $l$ apart given in Equation (11) by (Gift, 2010)

$$
\Delta t_{W E}=\frac{l}{c}+\frac{l v}{c^{2}}
$$

where, as before, $v$ is the speed of the Earth's surface at the particular latitude. Recall the light is travelling at speed $c$ in the ECI frame while the observer is moving on the rotating Earth at speed $v$ relative to the ECI frame. Light travel time $\Delta t_{W E}$ is also given by (Gift, 2010)

$$
\Delta t_{W E}=\frac{l}{c-v}=\frac{l}{c}+\frac{l v}{c^{2}}, v<<c
$$

Now consider the situation in Figure 4 where a vehicle at a position $A$ is moving at velocity $v_{C}$ relative to the surface $S$ (corresponding to light transmission at velocity $c$ relative to the ECI frame) and an observer a distance $L$ away at $B$ walking in the same direction at velocity $v$ relative to $S$ with $v<<v_{C}$ (corresponding to the observer a distance $l$ away moving on the rotating Earth at speed $v$ relative to the ECI frame).

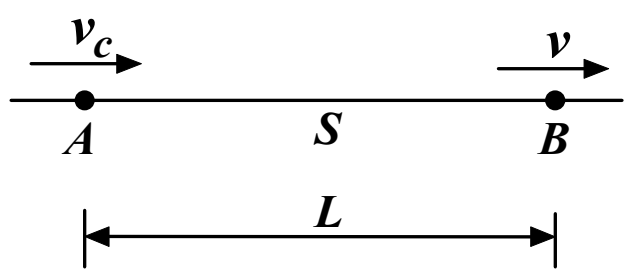

Figure 4. Vehicle at A and Observer at B both moving on Surface S

Then from classical mechanics the speed of the vehicle relative to the moving observer is given by $v_{C}-v$ and the time $T$ for the vehicle to catch up with the observer is given by

$$
T=\frac{L}{v_{C}-v}
$$

Expanding (43) and retaining only terms up to first-order this reduces to

$$
T=\frac{L}{v_{C}}+\frac{L v}{v_{C}^{2}}
$$

Comparing (44) and (41) it is clear that the situations are completely analogous. In the case of the moving vehicle however, (44) is not interpreted as the time $L / v_{C}$ for the vehicle to travel at speed $v_{C}$ relative to the observer with a "correction" $L v / v_{C}^{2}$ because of the movement of the observer. Instead the accepted and correct interpretation of (44) is this is the time $L /\left(v_{C}-v\right)$ for the vehicle to travel to the observer at speed $v_{C}-v$ relative to the observer. Similarly, in the GPS the interpretation of (41) as the time $l / c$ for the light to travel at speed $c$ relative to the observer on the rotating Earth with a "correction" $l v / c^{2}$ because of the rotating Earth is artificial and not tenable. Instead, as demonstrated above, the correct interpretation of (41) is that it is the time $l /(c-v)$ for the light to travel to the observer at speed $c-v$ relative to the observer.

\section{Discussion}

The results of this investigation reveal that the Langevin metric of general relativity which produces the rigorously verified CCIR clock synchronization algorithm, also yields noninvariant east-west light speeds $c \pm v$ in the frame of the rotating Earth. This is contrary to the light speed invariance principle postulated to hold in an approximately local inertial frame on the Earth and assumed true in the SI standard of length measurement. This light speed anisotropy has been experimentally confirmed by several researchers (Marmet, 2000; Kelly, 2005; Gift, 2010, 2011; Wang, 2000; Hatch, 2007; Sato, 2010) using the high-precision technology of the GPS. Moreover, by using elementary kinematics involving confirmed GPS time and arbitrary distance measurements, a more general expression $c_{R}=c-v \cos \theta$ for light speed relative to the surface of the rotating Earth was derived 
which easily yielded the CCIR clock synchronization algorithm. It was demonstrated that accurate clock synchronization uses $\int d \sigma^{\prime} /(c-v \cos \theta)$ and not $\int d \sigma^{\prime} / c$ since light speed in the frame of the rotating Earth is $c-$ $v \cos \theta$ and not $c$. The notion that the CCIR clock synchronization algorithm works since light travels at speed $c$ with the so-called "Sagnac correction" necessary because of the rotation of the Earth is an invalid interpretation that encourages belief in a constant light speed $c$.

Light transmission in the ECI frame at speed $c$ relative to this frame and modeled by the GPS range equation $\mid \bar{r}_{r}$ $\left(t_{r}\right)-\bar{r}_{s}\left(t_{s}\right) \mid=c\left(t_{r}-t_{s}\right)$ enabled a complete and accurate representation of the situation. Since the ECI frame moves with the Earth but does not rotate with it, an observer fixed on the surface of the Earth moves at speed $v$ relative to the ECI frame. Therefore the relative speed of light $c_{R}$ travelling in the ECI frame and observed on the surface of the rotating Earth was determined using the classical principles of relative velocity and shown to be $c_{R}$ $=c-v \cos \theta$. This is exactly the expression derived using the experimentally determined GPS light transmission time and simple kinematics.

To further illuminate the discussion, well-understood relative velocity occurring with low-speed vehicular motion was outlined and the equations shown to be completely analogous to those derived from the CCIR clock synchronization algorithm. The applicability of classical mechanics and relative speed to light transmission in the ECI frame was therefore revealed. These fully consistent theoretical and experimental results demonstrating light speed anisotropy in the Earth Centered Earth Fixed frame suggest that the principle of light speed constancy really is inapplicable in the rotating frame of the surface of the Earth. Such a view appears to be supported by Ashby who, despite his interpretation of the Sagnac Effect as a time correction, said (Ashby, 2002, p. 44) “...the principle of the constancy of $c$ cannot be applied in a rotating reference frame..."!

Selleri (1997) has shown that an unavoidable paradox arises when special relativity is applied to rotating platforms since the ratio $\rho$ of one-way east-west light speeds (both locally and globally) is $\rho \equiv \frac{c_{E W}}{c_{W E}}=\frac{1+v / c}{1-v / c} \neq 1$.

This is inconsistent with the light speed invariance postulate of the theory which requires $\rho=1$. Several attempts have been made to resolve this issue (Rizzi \& Tartaglia, 1998, 1999; Budden, 1998; Minguzzi, 2002; Ghosal et al., 2004) but Selleri in a later paper (Selleri, 2004a) reaffirmed the legitimacy of the finding $\rho \neq 1$. He sought a resolution of this problem (Selleri, 2010) by examining the most general set of space-time transformations which satisfy the experimentally observed conditions of constant two-way light speed and time dilation and which differ only by a clock synchronization parameter $e_{1}$. This allowed him to identify from among all possible transformations (including the Lorentz transformation) the single transformation that accords with all existing experimental data and for which the paradox does not arise. This turned out to be the Inertial transformation (Selleri, 2004b, 2011) corresponding to $e_{1}=0$ in the general set which has been independently confirmed (Gift, 2009). This transformation predicts one-way light speed on the rotating Earth as $c_{R}=\frac{c}{1+(v / c) \cos \theta}$ for which $\rho=\frac{1+v / c}{1-v / c}$ thereby resolving the paradox.

Using the anisotropic light speed $c_{R}=\frac{c}{1+(v / c) \cos \theta}$, Selleri (2010) calculated the time for an electromagnetic signal to travel between two points on the Earth via a geostationary satellite and thereby fully demonstrated the correction term $2 \omega_{E} \int d A_{Z}^{\prime} / c^{2}$. This result is exactly that obtained in this paper using light speed $c_{R}=c-v \cos \theta$. This equivalence occurs since light speed $c_{R}=\frac{c}{1+(v / c) \cos \theta}$ reduces to

$$
c_{R}=\frac{c}{1+(v / c) \cos \theta}=\frac{c(1-(v / c) \cos \theta)}{1-(v / c)^{2} \cos ^{2} \theta}=c-v \cos \theta, v<<c
$$

As a result of Selleri's derivation of the correction, he concluded "The procedure which we suggest to experimentalists is to avoid using a wrong velocity of light and correcting the result with an ad hoc term, but rather to use from the beginning the velocity of light $\left[c_{R}=\frac{c}{1+(v / c) \cos \theta}\right]$ of the inertial transformations."

Finally in the determination of light speed, synchronized clocks are required for the timing of a light pulse as it propagates between two fixed points. In this regard, the IEEE 1588 Standard for a Precision Clock Synchronization Protocol for Networked Measurement and Control Systems defines synchronized clocks as follows (IEEE-1588, 2002): "Two clocks are synchronized to a specified uncertainty if they have the same epoch and measurements of any time interval by both clocks differ by no more than the specified uncertainty. The timestamps generated by two synchronized clocks for the same event will differ by no more than the specified 
uncertainty." From this, clocks are synchronized if they indicate the same times for the same events and this is realized using a suitable synchronization procedure. Some researchers however employ certain other clock synchronization procedures that result in different modes of clock operation which they refer to as synchronized clocks. Light speeds determined using such clocks will show a dependence on these different synchronization schemes since differently synchronized clocks will measure different time intervals for the same light signal transmission. In fact virtually any speed, including $c$, can be obtained by suitably "synchronizing" the measuring clocks and Will (1992) has pointed out that "a particularly perverse choice of synchronization can make the apparent speed...infinite"! These "apparent" speeds are artificial and bear no relation to physical reality; they are meaningless.

A proper clock synchronization method must result in clocks that indicate the same times for the same events, precisely as occurs in the GPS. In this system it has been experimentally demonstrated that the use of any other clock synchronization algorithm would result in clock "de-synchronization" and hence major timing and navigational errors. All such alternatives are therefore invalid. Selleri addressed this issue in his consideration of the Sagnac Effect (Selleri, 2010). In addition to resolving the paradox by identifying the inertial transformation, he presented a new proof of absolute simultaneity by deducing the condition $e_{1}=0$ from the universal set of space-time transformations. In this way "absolute simultaneity is shown to be necessary in all theories" and like the GPS "it is not true that the synchronization procedure can be chosen freely" as many researchers seem to believe.

\section{Conclusion}

In their paper of 1988, Alley et al., (1988) expressed the view that "A fully successful theory of space, time, and gravity must be founded upon actual experience with the behaviour of clocks and the propagation of light pulses in gravitational fields and in accelerated frames of reference. The remarkable stability of current atomic clocks and the precision of short pulse laser ranging and time transfer systems now allow new types of experiments to be undertaken. These will allow the necessary experience to be gained." In keeping with this approach, the application of available GPS atomic clocks in simple time-measuring experiments discussed in this paper has enabled us to gain valuable information about the behaviour of light as it travels in the Earth Centred Earth Fixed frame. In particular the GPS has confirmed in Equations (24) and (25) and elsewhere that light travels faster West than East relative to the surface of the rotating Earth contradicting the widely accepted principle of light speed constancy. This is an objective fact validated by observational experience using synchronized clocks in the real-world time measurement of travelling light.

The Langevin metric used in general relativity to derive the successful CCIR clock synchronization algorithm was shown to also predict anisotropic east-west light speeds $c \pm v$. This prediction was supported using light travel times based on the GPS timing algorithm and simple kinematics as well as classical mechanics involving relative velocity in the ECI frame. Both methods yielded $c \pm v$ for east-west light travel between fixed points on the surface of the Earth. This was confirmed by actual experimental data from the GPS. Both approaches also produced the more general light speed expression $c-v \cos \theta$ for light travelling at an angle $\theta$ with respect to a line of latitude. This anisotropic result is consistent with Selleri's light speed $c_{R}=\frac{c}{1+(v / c) \cos \theta}$ predicted by his Inertial transformation but inconsistent with the light speed invariance principle requiring light speed $c_{R}=c$ which is assumed to hold locally in the otherwise non-inertial terrestrial frame. This expression $c_{R}=c-v \cos \theta$ for light speed relative to the surface of the rotating Earth was used to derive the CCIR clock synchronization algorithm that has been instrumental in the successful operation of the GPS. Here it was demonstrated that accurate clock synchronization uses $\int d \sigma^{\prime} /(c-v \cos \theta)$ and not $\int d \sigma^{\prime} / c$ because light speed in the frame of the rotating Earth is $c-v \cos \theta$ and not $c$.

Selleri has shown that the one-way light speed $c_{R}=\frac{c}{1+(v / c) \cos \theta}$ on the rotating Earth which reduces to $c_{R}=c$ $-v \cos \theta$ completely resolves the problem of the Sagnac correction. In an online dialogue involving authors of papers in the book on rotating frames (Rizzi \& Ruggiero, 2010, p. 399) he stated "My viewpoint about Sagnac-like corrections is that they arise from the anisotropic propagation of light in the Earth rotating frame. These "corrections" are fully accounted for if this anisotropy is properly taken into account, as I showed in my paper (Selleri, 2010, pp. 60-63)." In response to this comment Ashby conceded that "[Selleri's] viewpoint is admissible" but insisted that "it is not the only one." Selleri's sentiments are consonant with the results in this paper as well as with previous findings (Marmet, 2000; Kelly, 2005; Gift, 2010; Gift, 2011; Wang, 2000; Hatch, 2007; Sato, 2010).

Therefore these entirely consistent theoretical results and objective experimental facts provide incontrovertible 
evidence of light speed anisotropy in the Earth Centered Earth Fixed frame that deserves the attention of the scientific community. It was given attention long ago by Ives (1951) who after investigation argued that the principle of light speed constancy is not supported by "objective matters of fact" and is "untenable". Today however, there is rarely any mention of light speed anisotropy detection in the literature. In view of the ease with which light speed anisotropy is detected by the GPS, it is difficult to accept that the GPS experts have not themselves observed this anisotropy. One would have expected that with the availability of accurate synchronized clocks, there would by now be a widely accepted answer to the age-old question, what is the one-way speed of light travelling between two points fixed on the surface of the Earth? There is not and the answer $[c-v \cos \theta]$ presented in this paper and given also by Selleri (2010) is unfortunately not accepted by the community of scientists.

What is accepted by them despite the contradicting evidence is the idea that light always travels at a constant speed $c$ on the rotating Earth, a notion that they vigorously defend with a plethora of contrived arguments. Ashby's fallacious interpretation of the CCIR synchronization algorithm discussed in this paper in which he treats anisotropic light travel as constant light speed with a time correction is a good example of this. This researcher, the leading consultant on the GPS and its operation, has also incorrectly suggested in published work (Ashby, 1994) that light speed constancy in the ECI frame is an expression of the principle of light speed constancy. Wolf and Petit (1997) have perpetuated this misinformation by their false assertion that their light speed test conducted in the ECI frame where light speed is $c$ represents a test of light speed constancy postulated in all inertial frames. Perhaps the most egregious case is the uncritical acceptance by relativists of the many light speed tests yielding $c$ conducted in the non-inertial frame of the rotating Earth as valid confirmation of the principle of light speed constancy, while completely rejecting any test in the same rotating frame indicating light speed anisotropy on the grounds that the rotating frame is non-inertial! These arguments by the relativists which have no basis in reality must be vigorously challenged and ultimately rejected.

Light speed anisotropy, clearly detected by the GPS and manifested in the physical world as light travelling faster West than East, renders the principle of light speed constancy completely invalid on the rotating Earth. Such anisotropy leads to a definitive re-interpretation of the CCIR clock synchronization algorithm: The Sagnac effect should no longer be viewed as a necessary time correction in GPS operation arising from the rotation of the Earth since no such correction is necessary if the true speed of light is used in the travel time determination. Instead the Sagnac effect should be recognised as simply the time difference between separate light pulses arising from their different speeds as they travel in the rotating frame of the surface of the Earth. Ironically this Global Positioning System, hailed by the relativists as an outstanding example of the application of relativity theory, actually reveals the invalidity of the Einsteinian paradigm, criticism of which has been unceasing since its inception 100 years ago (Bethell, 2009). This invalidity we believe is the genesis of "The trouble with physics" (Smolin, 2006).

\section{References}

Adler, R., Bazin, M., \& Schiffer, M. (1975). Introduction to General Relativity. New York: McGraw Hill.

Allan, D. W., Weiss, M. A., \& Ashby, N. (1985). Around-the-World relativistic sagnac experiment. Science, 228, 69. http://dx.doi.org/10.1126/science.228.4695.69

Alley, C. O., Rayner, J. D., Steggerda, C. A., Mullendore, J. V., Small, L., \& Wagner, S. (1982). Time transfer between the goddard optical research facility and the u.s. naval observatory using 100 picosecond laser pulses. Proceedings of the $14^{\text {th }}$ Annual Precise Time and Time Interval (PTTI) Applications and Planning Meeting, 243-276.

Alley, C. O., Nelson, R. A., Shih, Y. H., Agnew, B. W., Bartolo, R. E., Broomfield, J. T., ... Wilcox, J. L. (1988). Differential comparison of the one-way speed of light in the east-west and west-east directions on the rotating Earth. Proceedings of the $20^{\text {th }}$ Annual Precise Time and Time Interval (PTTI) Applications and Planning Meeting, 261-284.

Antonini, P., Okhapkin, M., Goklu, E., \& Schiller, S. (2005). Test of constancy of speed of light with rotating $\begin{array}{llllll}\text { cryogenic } \text { optical } & \text { resonators. Physical Review } & \end{array}$ http://dx.doi.org/10.1103/PhysRevA.71.050101

Ashby, N. (1994). Relativity in the future of engineering. IEEE Transactions on Instrumentation and Measurement, 43, 505. http://dx.doi.org/10.1109/19.310159

Ashby, N. (2002). Relativity and the global positioning system. Physics Today, 55(5), 41-47. http://dx.doi.org/10.1063/1.1485583 
Ashby, N. (2003). Relativity in the global positioning system. Living Reviews in Relativity, 6, 1. Retrieved from http://www.emis.ams.org/journals/LRG/Articles/lrr-2003-1/download/lrr-2003-1BW.pdf

Ashby, N. (2006). Relativistic Effects in the Global Positioning System. Retrieved from http://www.aapt.org/doorway/tgru/articles/ashbyarticle.pdf accessed on October 24, 2012.

Ashby, N. (2010). The sagnac effect in the global positioning system, in relativity in rotating frames. London: Kluwer Academic Publishers.

Bethell, T. (2009). Questioning Einstein: Is relativity necessary? Colorado: Vales Lake Publishing.

Brillet, A. \& Hall, J. L. (1979). Improved laser test of the isotropy of space. Physical Review Letters, 42, 549. http://dx.doi.org/ 10.1103/PhysRevLett.42.549

Budden, T., (1998). Geometric simultaneity and the continuity of special relativity. Foundations of Physics Letters, 11, 343 .

Eisele, C., Nevsky, A., \& Schiller, S. (2009). Laboratory test of the isotropy of light propagation at the $10^{-17}$ level. Physical Review Letters, 103, 090401. http://dx.doi.org/ 10.1103/PhysRevLett.103.090401

Gagnon, D. R., Torr, D. G., Kolen, P. T., \& Chang, T. (1988). Guided-Wave measurement of the one-way speed of light. Physical Review A, 38, 1767. http://dx.doi.org/10.1103/PhysRevA.38.1767

Ghosal, S. K., Raychaudhuri, B., Chowdhury, A. K., \& Sarker, M. (2004). On the anisotropy of the speed of light on a rotating platform. Foundations of physics letters, $17,457$. http://dx.doi.org/10.1023/B:FOPL.0000042698.27763.0b

Gift, S. J. G. (2009). Separating equivalent space-time theories. Apeiron, 16, 408.

Gift, S. J. G. (2010). One-Way light speed measurement using the synchronized clocks of the global positioning system (GPS). Physics Essays, 23, 271. http://dx.doi.org/10.4006/1.3361840

Gift, S. J. G. (2011). One-Way light speed determination using the range measurement equation of the GPS. Applied Physics Research, 3(1), 110. http://dx.doi.org/10.5539/apr.v3n1p110

Hatch, R. R. (2007). A new theory of gravity: overcoming problems with general relativity. Physics Essays, 20, 83. http://dx.doi.org/10.4006/1.3073811

Hayden, H. C. (1991). Is the velocity of light isotropic in the frame of the rotating Earth? Physics Essays, 4, 361.

Hermann, S., Senger, A., Kovalchuk, E., Muller, H., \& Peters, A. (2005). Test of the isotropy of the speed of light using a continuously rotating optical resonator. Physical Review Letters, 95, 150401. http://dx.doi.org/10.1103/PhysRevLett.95.150401

IEEE-1588. (2002). Standard for a Precision Clock Synchronization Protocol for Networked Measurement and Control Systems.

IS-GPS-200E. (2010). Global positioning system wing (gpsw) systems engineering \& integration. Retrieved May 17, 2012 form http://www.gps.gov/technical/icwg/IS-GPS-200E.pdf

Ives, H. E. (1951). Revisions of the Lorentz transformations. Proceedings of the American Philosophical Society, 95, 125.

Kassner, K. (2012). Spatial geometry of the rotating disk and its non-rotating counterpart. American Journal of Physics, 80, 772. http://dx.doi.org/10.1119/1.4730925

Kelly, A. (2005). Challenging modern physics. Florida: BrownWalker Press.

Klauber, R. D. (1999). Comments regarding recent articles on relativistically rotating frames. American Journal of Physics, 67, 158. http://dx.doi.org/10.1119/1.19213

Krisher, T. P., Maleki, L., Lutes, G. F., Primas, L. E., Logan, R. T., Anderson, J. D., \& Will, C. M. (1990). Test of the isotropy of the one-way speed of light using hydrogen-maser frequency standards. Physical Review D, 42, 731. http://dx.doi.org/10.1103/PhysRevD.42.731

Landau, L., \& Lifschitz, E. M. (1975). The classical theory of fields (4th ed.). New York: Pergamon Press.

Marmet, P. (2000). The GPS and the constant velocity of light. Acta Scientiarum, 22, 1269.

Minguzzi, E. (2002). On the conventionality of simultaneity. Foundations of Physics Letters, 15, 153.

Moller, C. (1972). The theory of relativity (2nd ed.). Oxford, New York.

Muller, H., Herrmann, S., Braxmaier, C., \& Peters, A. (2003). Modern michelson-morley experiment using 
cryogenic optical resonators. Physical Review Letters, 91, 020401. http://dx.doi.org/10.1103/PhysRevLett.91.020401

Pascual-Sánchez, J.-F., San, Migue. A., \& Vicente, F. (2010). Isotropy of the velocity of light and the sagnac effect, in relativity in rotating frames. London: Kluwer Academic Publishers.

Riis, E., Lars-Ulrik, A. A., Bjerre, N., \& Poulsen, O. (1988). Test of the speed of light using fast-beam laser spectroscopy. Physical Review Letters, 60, 81. http://dx.doi.org/10.1103/PhysRevLett.60.81

Rindler, W. (2006). Relativity special, general and cosmological (2nd ed.). Oxford: Oxford University Press.

Rizzi, G., \& Tartaglia, A. (1998). Speed of light on rotating platforms. Foundations of Physics, 28, 1663. http://dx.doi.org/10.1023/A:1018893609690

Rizzi, G., \& Tartaglia, A. (1999). On local and global measurements of the speed of light on rotating platforms. Foundations of Physics Letters, 12, 179.

Rizzi, G., \& Ruggiero, M. L. (2002). Space geometry of rotating platforms: an operational approach. Foundations of Physics, 32, 1525. http://dx.doi.org/10.1023/A:1020427318877

Rizzi, G., \& Ruggiero, M. L. (2010). Relativity in rotating frames. London: Kluwer Academic Publishers.

Sato, M. (2010). The velocity of electromagnetic wave is observed differently depending on the observer's velocity. Physics Essays, 23, 405. http://dx.doi.org/10.4006/1.3452472

Selleri, F. (1997). Noninvariant one-way speed of light and locally equivalent reference frames. Foundations of Physics Letters, 10, 73. http://dx.doi.org/10.1007/BF02764121

Selleri, F. (2004a). Curing one discontinuity with another: comment on S. K. Ghosal et al., "On the anisotropy of the speed of light on a rotating platform". Foundations of Physics Letters, 17, 599. http://dx.doi.org/10.1007/s10702-004-0906-5

Selleri, F. (2004b). Recovering the Lorentz ether. Apeiron, 11, 246.

Selleri, F. (2010). In G. Rizzi \& M. L. Ruggiero (Eds.), Sagnac effect: end of the mystery, in relativity in rotating frames. London: Kluwer Academic Publishers.

Selleri, F. (2011). La Relativita Debole. Milano: Edizioni Melquiades.

Smolin, L. (2006). The trouble with physics. New York: Houghton Mifflin.

Tartaglia, A., \& Ruggiero, M. L. (2002). Angular momentum effects in michelson-morley type experiments. General Relativity and Gravitation, 34, 1371. http://dx.doi.org/10.1023/A:1020022717216

Wang, R. (2000). Successful GPS operations contradict the two principles of special relativity and imply a new way for inertial navigation-measuring speed directly. Proceedings of the IAIN World Congress and the 56th Annual Meeting of the Institute of Navigation, San Diego, CA.

Will, C. M. (1992). Clock synchronization and isotropy of the one-way speed of light. Physical Review D, 45, 403. http://dx.doi.org/10.1103/PhysRevD.45.403

Wolf, P., \& Petit, G. (1997). Satellite test of special relativity using the global positioning system. Physical Review A, 56, 4405. http://dx.doi.org/10.1103/PhysRevA.56.4405

Yilmaz, H. (1976). Physical foundations of the new theory of gravitation. Annals of Physics, 101, 413. http://dx.doi.org/10.1016/0003-4916(76)90018-X

Yilmaz, H. (1982). Relativity and quantum mechanics. International Journal of Theoretical Physics, 21, 871. http://dx.doi.org/10.1007/BF01856878

Zhang, Y. Z. (1997). Special relativity and its experimental foundations. Singapore: World Scientific.

\section{Copyrights}

Copyright for this article is retained by the author(s), with first publication rights granted to the journal.

This is an open-access article distributed under the terms and conditions of the Creative Commons Attribution license (http://creativecommons.org/licenses/by/3.0/). 